\title{
An Improved experimental databank of transferable multipolar atom models - ELMAM2. Construction details and applications
}

Sławomir Domagała, Bertrand Fournier, Dorothee Liebschner, Benoît Guillot ${ }^{a}$ \& Christian Jelsch ${ }^{*}$

Laboratoire de Cristallographie, Résonance Magnétique et Modélisations (CRM2), CNRS, UMR 7036, Institut Jean Barriol, Faculté des Sciences et Techniques, Nancy Université, BP 70239, 54506 Vandoeuvre-lès-Nancy CEDEX, France E-mail: christian.jelsch@crm2.uhp-nancy.fr

\section{SUPPORTING INFORMATION Supplementary Tables 1S-6S Supplementary Figures 1S}




\section{Supplementary Tables}

Table 1S. List of crystal structures used for building the ELMAM2 database.

\begin{tabular}{|c|c|c|c|c|}
\hline $\mathrm{Nr}$ & Abbreviation & Name, formula & $\begin{array}{l}\text { Data type, } \\
\text { temp }\end{array}$ & Reference \\
\hline 1 & ala & $\begin{array}{l}\text { L-Alanine, } \\
\mathrm{C}_{3} \mathrm{H}_{7} \mathrm{NO}_{2}\end{array}$ & X-Ray, Mo, 23K & Destro et al., 1988 \\
\hline 2 & enkephalin & $\begin{array}{l}\text { Leu-Enkephalin Trihydrate, } \\
\mathrm{C}_{28} \mathrm{H}_{37} \mathrm{~N}_{5} \mathrm{O}_{7} 3 \mathrm{H}_{2} \mathrm{O}\end{array}$ & X-Ray, Mo, $100 \mathrm{~K}$ & Wiest et al., 1994 \\
\hline 3 & Argphos & $\begin{array}{l}\text { L-Arginine Phosphate } \\
\text { Monohydrate, } \mathrm{C}_{6} \mathrm{H}_{15} \mathrm{~N}_{4} \mathrm{O}_{2}{ }^{+} \mathrm{H}_{2} \mathrm{PO}_{4}^{-} \\
\mathrm{H}_{2} \mathrm{O}\end{array}$ & X-Ray, Mo, $130 \mathrm{~K}$ & Espinosa et al., 1996 \\
\hline 4 & oxalic & $\begin{array}{l}\text { Oxalic acid, } \\
\mathrm{C}_{2} \mathrm{H}_{2} \mathrm{O}_{4}\end{array}$ & X-Ray, Mo, 100K & Martin et al., 1998 \\
\hline 5 & his & $\begin{array}{l}\text { DL-Histidine, } \\
\mathrm{C}_{6} \mathrm{H}_{9} \mathrm{~N}_{3} \mathrm{O}_{2}\end{array}$ & X-Ray, Mo, $110 \mathrm{~K}$ & Coppens et al., 1999 \\
\hline 6 & actyr & $\begin{array}{l}\mathrm{N} \text {-acetyl-L-tyrosine ethyl ester } \\
\text { monohydrate, } \\
\mathrm{C}_{13} \mathrm{H}_{19} \mathrm{NO}_{5}\end{array}$ & X-Ray, Mo, $110 \mathrm{~K}$ & Dahaoui et al., 1999 \\
\hline 7 & glythr & $\begin{array}{l}\text { Glycyl-L-threonine dihydrate, } \\
\mathrm{C}_{6} \mathrm{H}_{12} \mathrm{~N}_{2} \mathrm{O}_{4} 2 \mathrm{H}_{2} \mathrm{O}\end{array}$ & X-Ray, Mo, $110 \mathrm{~K}$ & Benabicha et al., 2000 \\
\hline 8 & GlyAsp & $\begin{array}{l}\text { glycyl-aspartic acid dihydrate, } \\
\mathrm{C}_{6} \mathrm{H}_{10} \mathrm{~N}_{2} \mathrm{O}_{5} 2 \mathrm{H}_{2} \mathrm{O}\end{array}$ & X-Ray, Mo, $123 \mathrm{~K}$ & $\begin{array}{l}\text { Pichon-Pesme et al., } \\
2000\end{array}$ \\
\hline 9 & TyrGlyGly & $\begin{array}{l}\text { Tyrosyl-glycyl-glycine } \\
\text { monohydrate, } \mathrm{C}_{13} \mathrm{H}_{17} \mathrm{~N}_{3} \mathrm{O}_{5} \mathrm{H}_{2} \mathrm{O}\end{array}$ & X-Ray, Mo, 123K & $\begin{array}{l}\text { Pichon-Pesme et al., } \\
2000\end{array}$ \\
\hline 10 & panb & $\begin{array}{l}p \text {-amino- } p^{\prime} \text {-nitrobiphenyl, } \\
\mathrm{C}_{12} \mathrm{H}_{10} \mathrm{~N}_{2} \mathrm{O}_{2}\end{array}$ & X-Ray, Mo, 20K & Volkov et al., 2000 \\
\hline 11 & ureaphos & urea-phosphoric acid, $\mathrm{CH}_{7} \mathrm{~N}_{2} \mathrm{O}_{5} \mathrm{P}$ & X-Ray, Mo, $100 \mathrm{~K}$ & Rodrigues et al., 2001 \\
\hline 12 & NTO & $\begin{array}{l}\text { 5-nitro-2,4-dihydro-3H-1,2,4- } \\
\text { triazol-3-one, - } \mathrm{NTO}, \mathrm{C}_{2} \mathrm{H}_{2} \mathrm{~N}_{4} \mathrm{O}_{3}\end{array}$ & X-Ray, Ag, 100K & Zhurova et al., 2001 \\
\hline 13 & cycloproala & $\begin{array}{l}\text { cyclo-(D,L-Pro })_{2}-(\text { L-Ala })_{4} \\
\text { monohydrate, } \\
\mathrm{C}_{22} \mathrm{H}_{34} \mathrm{~N}_{6} \mathrm{O}_{6} \mathrm{H}_{2} \mathrm{O}\end{array}$ & $\begin{array}{l}\text { Synchrotron, } \\
0.5583 \AA \text { A, 100K }\end{array}$ & Dittrich et al., 2002 \\
\hline 14 & $\begin{array}{l}\text { Thiocoumarin } \\
2\end{array}$ & $\begin{array}{l}2 \mathrm{H} \text {-chromene-2-thione, }(2- \\
\text { thiocomarin) } \\
\mathrm{C}_{9} \mathrm{H}_{6} \mathrm{OS}\end{array}$ & X-Ray, Mo, 90K & Munshi et al., 2002 \\
\hline 15 & HPPM & $\begin{array}{l}\text { hydrogen-[(2,4-diaminopyrimidin- } \\
\text { 1-io)methyl]-phosphonate } \\
\text { monohydrate, } \\
\mathrm{C}_{5} \mathrm{H}_{9} \mathrm{~N}_{4} \mathrm{O}_{3} \mathrm{P} \mathrm{H}_{2} \mathrm{O}\end{array}$ & X-Ray, Mo, 105K & Slouf et al., 2002 \\
\hline 16 & $\mathrm{BIGH}$ & $\begin{array}{l}\text { Biguanidinium Dinitramide, } \\
\mathrm{C}_{2} \mathrm{H}_{8} \mathrm{O}_{4} \mathrm{~N}_{8}\end{array}$ & X-Ray, Mo, 90K & Zhurova et al., 2002 \\
\hline 17 & $\mathrm{BIGH} 2$ & $\begin{array}{l}\text { Biguanidinium Bis-Dinitramide, } \\
\mathrm{C}_{2} \mathrm{H}_{9} \mathrm{O}_{8} \mathrm{~N}_{11}\end{array}$ & X-Ray, Mo, 90K & Zhurova et al., 2002 \\
\hline 18 & schiff1 & $\begin{array}{l}\text { dianil of 2-hydroxy-5-methyl- } \\
\text { isophth-aldehyde, } \\
\mathrm{C}_{21} \mathrm{H}_{18} \mathrm{O}_{1} \mathrm{~N}_{2}\end{array}$ & X-Ray, Mo, $100 \mathrm{~K}$ & Dominiak et al., 2003 \\
\hline 19 & schiff2 & $\begin{array}{l}\text { 3,5-dinitro- } \mathrm{N} \text { - } \\
\text { salicylidenoethylamine, } \\
\mathrm{C}_{9} \mathrm{H}_{9} \mathrm{O}_{5} \mathrm{~N}_{3}\end{array}$ & X-Ray, Mo, $100 \mathrm{~K}$ & Dominiak et al., 2003 \\
\hline 20 & schiff3 & $\begin{array}{l}\text { 3-nitro- } N \text { - } \\
\text { salicylidenocyclohexylamine, } \\
\mathrm{C}_{13} \mathrm{H}_{16} \mathrm{O}_{3} \mathrm{~N}_{2}\end{array}$ & X-Ray, Mo, $100 \mathrm{~K}$ & Dominiak et al., 2003 \\
\hline 21 & nad & $\begin{array}{l}\mathrm{NAD}+, \beta \text {-Nicotinamide Adenine } \\
\text { Dinucleotide, } \\
\mathrm{C}_{21} \mathrm{H}_{27} \mathrm{~N}_{7} \mathrm{O}_{14} \mathrm{P}_{2} 4 \mathrm{H}_{2} \mathrm{O}\end{array}$ & X-Ray, Mo, 100K & Guillot et al., 2003 \\
\hline
\end{tabular}




\begin{tabular}{|c|c|c|c|c|}
\hline 22 & TFT & $\begin{array}{l}\text { Tetrafluoroterephthalonitrile, } \\
\mathrm{C}_{8} \mathrm{~N}_{2} \mathrm{~F}_{4}\end{array}$ & $\begin{array}{l}\text { X-Ray, Mo, } \\
122.4 \mathrm{~K}\end{array}$ & Sørensen et al., 2003 \\
\hline 23 & urea & $\begin{array}{l}\text { Urea, } \\
\mathrm{CH}_{4} \mathrm{~N}_{2} \mathrm{O}\end{array}$ & $\begin{array}{l}\text { Synchrotron, } \\
0.5996 \AA \text { 足 } 123 \mathrm{~K}\end{array}$ & Birkedal et al., 2004 \\
\hline 24 & busulfan & $\begin{array}{l}\text { 1,4-butanediol-dimethylsulfonate, } \\
\mathrm{C}_{6} \mathrm{H}_{14} \mathrm{O}_{6} \mathrm{~S}_{2}\end{array}$ & X-Ray, Mo, 100K & Ghermani et al., 2004 \\
\hline 25 & barba & $\begin{array}{l}\text { 2,4,6,8-tetraphenylbarbaralane, } \\
\mathrm{C}_{33} \mathrm{H}_{26}\end{array}$ & $\begin{array}{l}\text { Synchrotron, } \\
0.56 \AA \AA 110 \mathrm{~K}\end{array}$ & Luger et al., 2004 \\
\hline 26 & xylitol & $\begin{array}{l}\text { xylitol, } \\
\mathrm{C}_{5} \mathrm{H}_{12} \mathrm{O}_{5}\end{array}$ & $\begin{array}{l}\text { X-Ray, Mo, } \\
122.4 \mathrm{~K}\end{array}$ & Madsen et al., 2004 \\
\hline 27 & famotidine $A$ & $\begin{array}{l}\text { Famotidine (polymorph A), } \\
\mathrm{C}_{8} \mathrm{H}_{15} \mathrm{~N}_{7} \mathrm{O}_{2} \mathrm{~S}_{3}\end{array}$ & X-Ray, Mo, 100K & Overgaard et al., 2004 \\
\hline 28 & famotidineB & $\begin{array}{l}\text { Famotidine (polymorph B), } \\
\mathrm{C}_{8} \mathrm{H}_{15} \mathrm{~N}_{7} \mathrm{O}_{2} \mathrm{~S}_{3}\end{array}$ & X-Ray, Mo, 100K & Overgaard et al., 2004 \\
\hline 29 & $\operatorname{trp}$ & $\begin{array}{l}\text { L-tryptophan formic acid, } \\
\mathrm{C}_{11} \mathrm{H}_{12} \mathrm{~N}_{2} \mathrm{O}_{2} \mathrm{CH}_{2} \mathrm{O}_{2}\end{array}$ & $\begin{array}{l}\text { Synchrotron, } \\
0.54 \AA \text { A } 100 \mathrm{~K}\end{array}$ & Scheins et al., 2004 \\
\hline 30 & coumarin & $\begin{array}{l}\text { 2H-chromene-2-one, } \\
\mathrm{C}_{9} \mathrm{H}_{6} \mathrm{O}_{2}\end{array}$ & X-Ray, Mo, 90K & Munshi et al., 2005 \\
\hline 31 & $\begin{array}{c}\text { Thiocoumarin } \\
1\end{array}$ & $\begin{array}{l}\text { 1-thiocumarin, } \\
\mathrm{C}_{9} \mathrm{H}_{6} \mathrm{OS}\end{array}$ & X-Ray, Mo, 90K & Munshi et al., 2005 \\
\hline 32 & $\begin{array}{l}\text { pentaerythrit } \\
\text { ol }\end{array}$ & $\begin{array}{l}\text { Pentaerythritol, } \\
\mathrm{C}_{5} \mathrm{H}_{12} \mathrm{O}_{4}\end{array}$ & X-Ray, Mo, $15 \mathrm{~K}$ & Zhurov et al., 2005 \\
\hline 33 & accoumarin $A$ & $\begin{array}{l}\text { 3-Acetylcoumarin, (polymorph A), } \\
\mathrm{C}_{11} \mathrm{H}_{8} \mathrm{O}_{3}\end{array}$ & X-Ray, Mo, 90K & Munshi et al., 2006a \\
\hline 34 & accoumarin B & $\begin{array}{l}\text { 3-Acetylcoumarin, (polymorph B), } \\
\mathrm{C}_{11} \mathrm{H}_{8} \mathrm{O}_{3}\end{array}$ & X-Ray, Mo, 90K & Munshi et al., 2006a \\
\hline 35 & cytosine_hyd & $\begin{array}{l}\text { cytosine monohydrate, } \\
\mathrm{C}_{4} \mathrm{H}_{5} \mathrm{~N}_{3} \mathrm{O} \mathrm{H}_{2} \mathrm{O}\end{array}$ & X-Ray, Mo, 90K & Munshi et al., 2006b \\
\hline 36 & salicylic & $\begin{array}{l}\text { salicylic acid, } \\
\mathrm{C}_{7} \mathrm{H}_{6} \mathrm{O}_{3}\end{array}$ & X-Ray, Mo, 90K & Munshi et al., 2006b \\
\hline 37 & thiosemicarb & $\begin{array}{l}\text { 1-formyl-3- } \\
\text { thiosemicarbazide, } \mathrm{C}_{2} \mathrm{H}_{5} \mathrm{~N}_{3} \mathrm{OS}\end{array}$ & X-Ray, Mo, 90K & Munshi et al., 2006c \\
\hline 38 & thiouracil & 2-thiouracil, $\mathrm{C}_{4} \mathrm{H}_{4} \mathrm{~N}_{2} \mathrm{OS}$ & X-Ray, Mo, 90K & Munshi et al., 2006b \\
\hline 39 & stilbene & Dimethylstilbene & X-Ray, 16K & Ogawa et al., 2006 \\
\hline 40 & estradiol & $\begin{array}{l}17 \beta \text {-Estradiol Urea, } \\
\mathrm{C}_{18} \mathrm{H}_{24} \mathrm{O}_{2} \mathrm{CH}_{4} \mathrm{~N}_{2} \mathrm{O}\end{array}$ & X-Ray, Mo, 100K & Parrish et al., 2006 \\
\hline 41 & estrone & $\begin{array}{l}\text { 3-hydroxy-1,3,5- } \\
\text { (10)-estratrien-17-one, } \\
\mathrm{C}_{18} \mathrm{H}_{22} \mathrm{O}_{2}\end{array}$ & X-Ray, Mo, 20K & Zhurova et al., 2006 \\
\hline 42 & $\begin{array}{c}\text { diazapentalen } \\
\mathrm{e}\end{array}$ & $\begin{array}{l}\text { 1,3,4-trinitro-7,8-diazapentalene, } \\
\mathrm{C}_{6} \mathrm{H}_{3} \mathrm{~N}_{5} \mathrm{O}_{6}\end{array}$ & X-Ray, Ag, 90K & Chen et al., 2007 \\
\hline 43 & sarcosine & $\begin{array}{l}\text { Sarcosine, } \\
\mathrm{C}_{3} \mathrm{H}_{7} \mathrm{NO}_{2}\end{array}$ & X-Ray, Mo, 100K & Dittrich et al., 2007 \\
\hline 44 & alaproala & $\begin{array}{l}\text { L-alanyl-L-prolyl-L-alanine hydrate, } \\
\mathrm{C}_{11} \mathrm{H}_{19} \mathrm{~N}_{3} \mathrm{O}_{4} \mathrm{H}_{2} \mathrm{O}\end{array}$ & $\begin{array}{l}\text { Synchrotron, } \\
100 \mathrm{~K}\end{array}$ & Kalinowski et al., 2007 \\
\hline 45 & ornithine & $\begin{array}{l}\text { L-ornithine hydrochloride, } \\
\mathrm{C}_{5} \mathrm{H}_{13} \mathrm{~N}_{2} \mathrm{O}_{2} \mathrm{Cl}\end{array}$ & X-Ray, Mo, 100K & Dittrich et al., 2007 \\
\hline 46 & thymidine & $\begin{array}{l}\text { Thymidine, } \\
\mathrm{C}_{10} \mathrm{H}_{14} \mathrm{~N}_{2} \mathrm{O}_{5}\end{array}$ & X-Ray, Mo, 20K & Hübschle et al., 2008 \\
\hline 47 & thiouronium & $\begin{array}{l}\text { 2-(indol-3-yl)- } \\
\text { 1,1,3,3-tetramethylthiouronium } \\
\text { nitrate, } \\
\mathrm{C}_{13} \mathrm{H}_{18} \mathrm{~N}_{3} \mathrm{~S}^{+} \mathrm{NO}_{3}^{-}\end{array}$ & X-Ray, Mo, 110K & Lutz et al., 2008 \\
\hline 48 & FOX-7 & $\begin{array}{l}\text { 1,1- } \\
\text { diamino-2,2-dinitroethylene, } \\
\mathrm{C}_{2} \mathrm{H}_{4} \mathrm{~N}_{4} \mathrm{O}_{4}\end{array}$ & $\begin{array}{l}\text { Synchrotron, } \\
0.503 \AA, 100 \mathrm{~K}\end{array}$ & Meents et al., 2008 \\
\hline 49 & $\mathrm{RhPCl}$ & $\begin{array}{l}{[\mathrm{Rh}(\mathrm{C} 7 \mathrm{H} 8)(\mathrm{PPh} 3) \mathrm{Cl}]} \\
\mathrm{C}_{25} \mathrm{H}_{23} \mathrm{ClPRh}\end{array}$ & X-Ray, Mo, 100K & Sparkes et al., 2008 \\
\hline 50 & paracetamol & $\begin{array}{l}\text { paracetamol, } \\
\mathrm{C}_{8} \mathrm{H}_{9} \mathrm{NO}_{2}\end{array}$ & X-Ray, Mo, 100K & Bouhmaida et al., 2009 \\
\hline
\end{tabular}




\begin{tabular}{|c|c|c|c|c|}
\hline 51 & $16 \mathrm{Ni}$ & $\begin{array}{l}{[3,11 \text {-Bis(methoxycarbonyl)- }} \\
1,5,9,13 \text {-tetraazacyclohexadeca- } \\
1,3,9,11- \\
\left.\text { tetraenato-(2-)- } \kappa^{4} \mathrm{~N}\right] \text { nickel(II), } \\
\mathrm{C}_{16} \mathrm{H}_{22} \mathrm{~N}_{4} \mathrm{O}_{4} \mathrm{Ni}\end{array}$ & X-Ray, Mo, 100K & Domagała et al., 2009 \\
\hline 52 & fidarestat & $\begin{array}{l}\text { Fidarestat, } \\
\mathrm{C}_{12} \mathrm{H}_{10} \mathrm{~N}_{3} \mathrm{O}_{4} \mathrm{~F}\end{array}$ & $\begin{array}{l}\text { Synchrotron, } \\
0.450 \AA, 100 \mathrm{~K}\end{array}$ & Fournier et al., 2009 \\
\hline 53 & coumcarb & $\begin{array}{l}\text { coumarin-3-carboxylic } \\
\text { acid, } \\
\mathrm{C}_{10} \mathrm{H}_{6} \mathrm{O}_{4}\end{array}$ & X-Ray, Mo, $100 \mathrm{~K}$ & Howard et al., 2009 \\
\hline 54 & transcin & $\begin{array}{l}\text { trans-cinnamic acid, } \\
\mathrm{C}_{9} \mathrm{H}_{8} \mathrm{O}_{2}\end{array}$ & X-Ray, Mo, 100K & Howard et al., 2009 \\
\hline
\end{tabular}


Table 2S. Schematic representation of the selected, available atom-types in the ELMAM2.

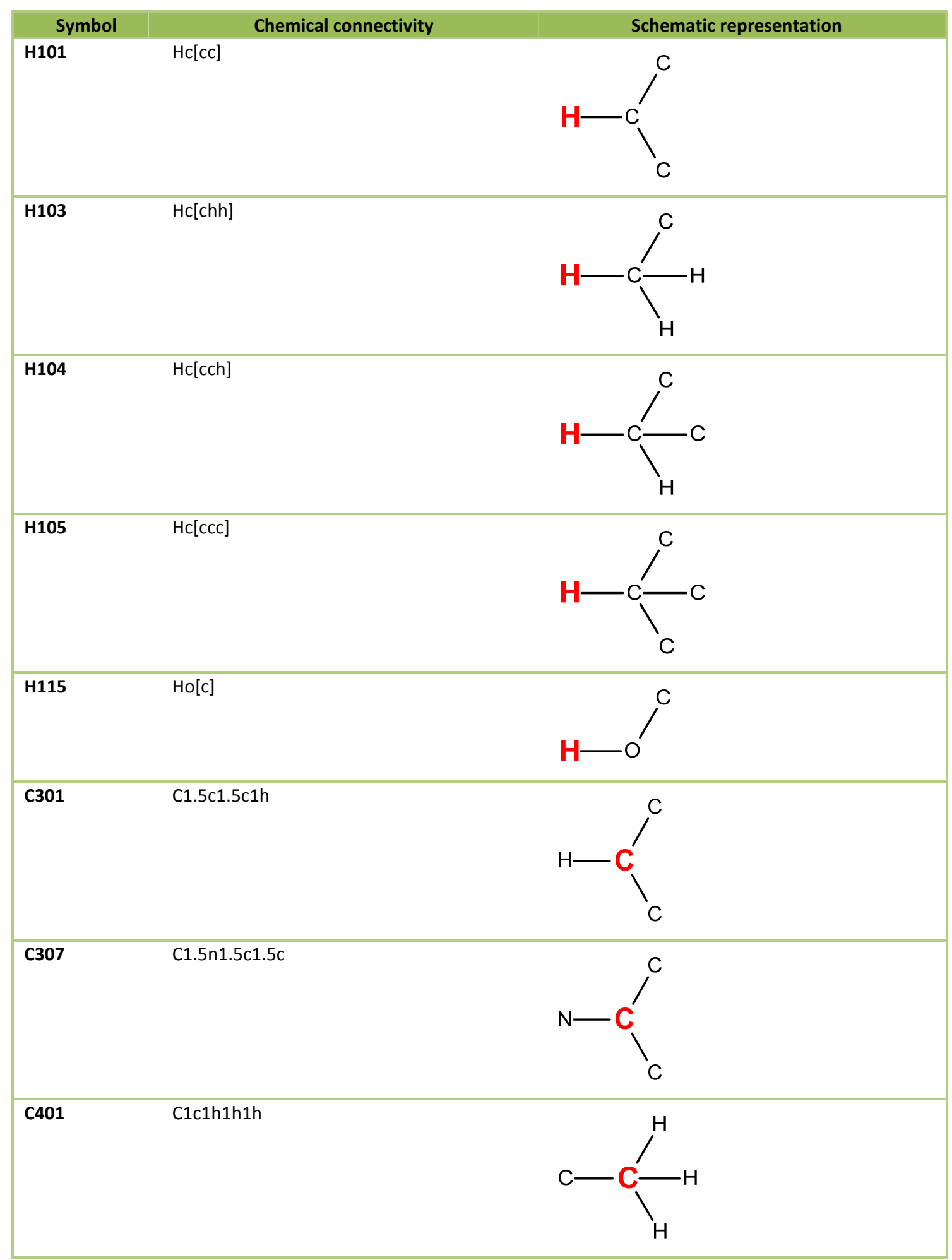




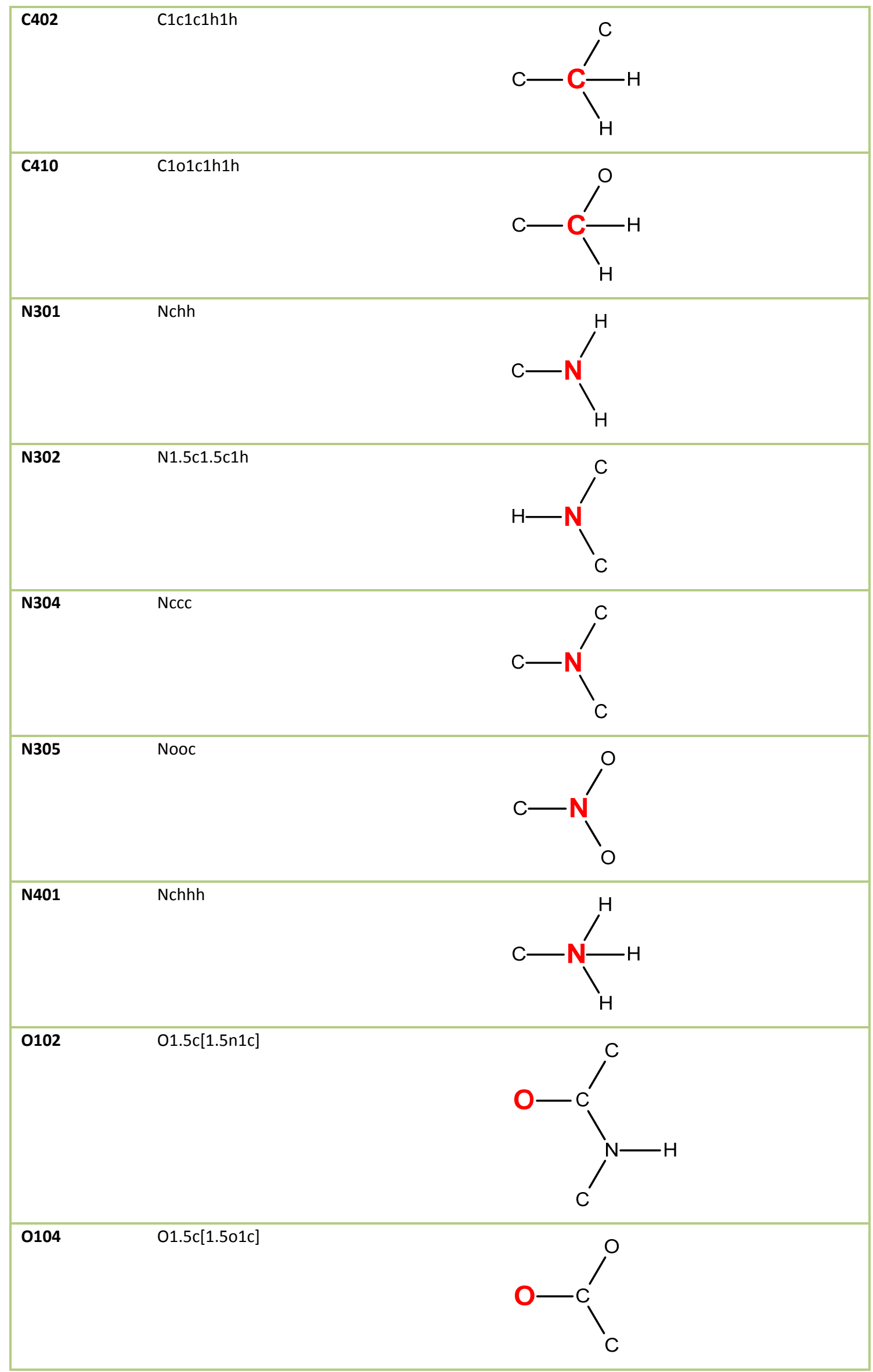




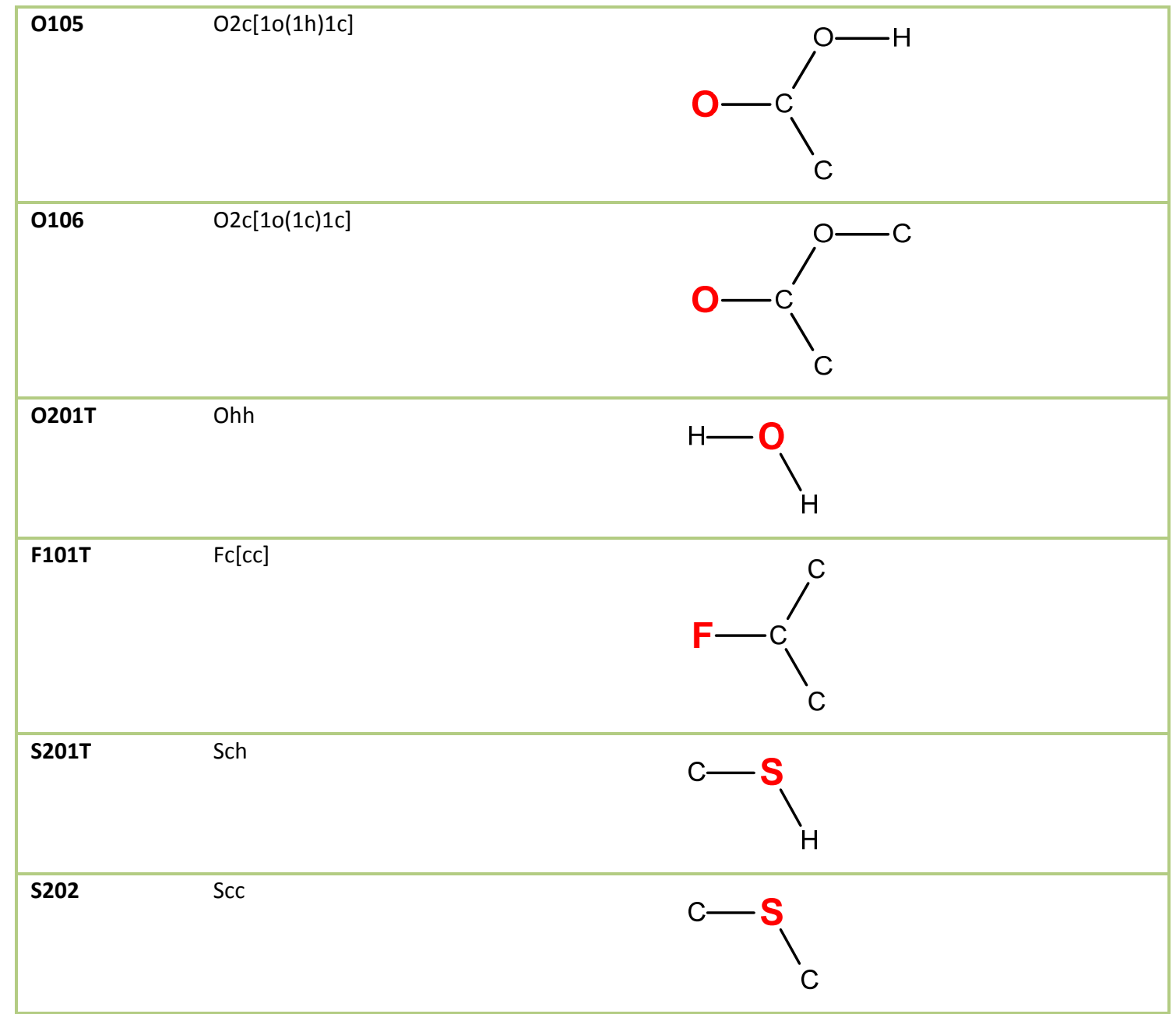

The connectivity is shown without the bond orders. In total 68 atom-types are available; $16 \mathrm{H} ; 31 \mathrm{C}$; $7 \mathrm{~N} ; 11 \mathrm{O} ; 1 \mathrm{~F} ; 2 \mathrm{~S}$ 
Table 3S. Benchmarked crystal structures.

\begin{tabular}{|c|c|c|c|c|}
\hline Compound & Structure details & $\begin{array}{l}\text { Theoretical } \\
\text { calculations }\end{array}$ & Database transfer details & Reference \\
\hline Glycine & $\begin{array}{l}\text { high resolution X- } \\
\text { Ray, } 23 \mathrm{~K}\end{array}$ & Single Point & $\begin{array}{l}\text { AMBER (a mixture of } \mathrm{C} \text { - } \\
\text { terminal glycine, } \mathrm{N} \text {-terminal } \\
\text { glycine and bulk glycine } \\
\text { used for } \mathrm{CA}, \mathrm{HA} 1 \text { and } \mathrm{HA} 2 \\
\text { point charges) } \\
\text { ELMAM } \\
\text { ELMAM2 }\end{array}$ & $\begin{array}{l}\text { Destro, R.; Roversi, P.; } \\
\text { Barzaghi, M.; Marsh, R. } \\
\text { E. J. Phys. Chem. A, } \\
\text { 2000, 104, 1047-1054. }\end{array}$ \\
\hline $\begin{array}{l}\text { AlaGlyAla } \\
\text { anhydrous }\end{array}$ & X-Ray, 293K & $\begin{array}{l}\text { Single Point, } \\
\mathrm{X} \text {-H distances } \\
\text { extended to } \\
\text { average } \\
\text { neutron } \\
\text { values }\end{array}$ & $\begin{array}{l}\text { AMBER } \\
\text { ELMAM } \\
\text { ELMAM2 }\end{array}$ & $\begin{array}{l}\text { Sreekanta Padiyar, G.; } \\
\text { Parthasarathy Seshadri, } \\
\text { T. Acta Crystallogr., Sect. } \\
\text { C, 1996, 52, 1693-1695. }\end{array}$ \\
\hline $\begin{array}{l}\text { AlaGlyAla } \\
\text { monohydrate }\end{array}$ & X-Ray, 20K & $\begin{array}{l}\text { Single Point, } \\
\mathrm{X}-\mathrm{H} \text { distances } \\
\text { extended to } \\
\text { average } \\
\text { neutron } \\
\text { values }\end{array}$ & $\begin{array}{l}\text { AMBER } \\
\text { ELMAM } \\
\text { ELMAM2 }\end{array}$ & $\begin{array}{l}\text { Förster, D.; } \\
\text { Messerschmidt, M.; } \\
\text { Luger, P. Acta } \\
\text { Crystallogr., Sect. C, } \\
\text { 2005, 61, 0420-0421. }\end{array}$ \\
\hline $\begin{array}{l}\text { AlaProAla } \\
\text { monohydrate }\end{array}$ & $\begin{array}{l}\text { high resolution, } \mathrm{X} \text { - } \\
\text { Ray, } 100 \mathrm{~K}\end{array}$ & Single Point & $\begin{array}{l}\text { AMBER } \\
\text { ELMAM } \\
\text { ELMAM2 }\end{array}$ & $\begin{array}{l}\text { Kalinowski, R.; Dittrich, } \\
\text { B.; Hübschle, C. B.; } \\
\text { Paulmann, C.; Luger, P. } \\
\text { Acta Crystallogr., Sect. } \\
\text { B, 2007, 63, } \\
\text { 753-767. }\end{array}$ \\
\hline $\begin{array}{l}\text { AlaTyrAla } \\
\text { ethanol }\end{array}$ & X-Ray, 20K & $\begin{array}{l}\text { Single Point, } \\
\mathrm{X}-\mathrm{H} \text { distances } \\
\text { extended to } \\
\text { average } \\
\text { neutron } \\
\text { values }\end{array}$ & $\begin{array}{l}\text { AMBER } \\
\text { ELMAM } \\
\text { ELMAM2 }\end{array}$ & $\begin{array}{l}\text { Chęcińska, L.; Förster, } \\
\text { D.; Morgenroth, W.; } \\
\text { Luger, P. Crystallogr., } \\
\text { Sect. C, 2006, 62, o454- } \\
\text { o457. }\end{array}$ \\
\hline benzene & Neutron, $138 \mathrm{~K}$ & Single Point & $\begin{array}{l}\text { ELMAM (tyrosine aromatic } \\
\text { ring used to mimic atom- } \\
\text { types) } \\
\text { ELMAM2 }\end{array}$ & $\begin{array}{l}\text { Bacon, G. E.; Curry, N. } \\
\text { A.; Wilson, S. A. Proc. R. } \\
\text { Soc. London Ser. A, } \\
\text { 1964, 279, 98-110. }\end{array}$ \\
\hline catechol & X-Ray, 100K & $\begin{array}{l}\text { Full } \\
\text { Optimization }\end{array}$ & $\begin{array}{l}\text { ELMAM (tyrosine aromatic } \\
\text { ring used to mimic atom- } \\
\text { types) }\end{array}$ & $\begin{array}{l}\text { Fronczek, F. R.; Kim, K. } \\
\text { K.; Strongin, R. M.; Priv. } \\
\text { Comm., } 2002 .\end{array}$ \\
\hline
\end{tabular}




\begin{tabular}{|c|c|c|c|c|}
\hline & & & ELMAM2 & (CATCOL13) \\
\hline resorcinol & Neutron, 298K & Single Point & $\begin{array}{l}\text { ELMAM (tyrosine aromatic } \\
\text { ring used to mimic atom- } \\
\text { types) } \\
\text { ELMAM2 }\end{array}$ & $\begin{array}{l}\text { Bacon, G. E.; Jude R. J. } \\
\text { Z.Kristallogr., } \\
\text { Kristallgeom., } \\
\text { Kristallphys., } \\
\text { Kristallchem. 1973, 138, } \\
\text { 19-40. }\end{array}$ \\
\hline $\begin{array}{l}p \text {-nitrophenol } \\
\alpha \text {-form }\end{array}$ & $\begin{array}{l}\text { high resolution X- } \\
\text { Ray, } 110 \mathrm{~K}\end{array}$ & Single Point & $\begin{array}{l}\text { ELMAM (tyrosine aromatic } \\
\text { ring used to mimic atom- } \\
\text { types) } \\
\text { ELMAM2 }\end{array}$ & $\begin{array}{l}\text { Kulkarni, G. U.; } \\
\text { Kumaradhas, P.; Rao, C. } \\
\text { N. R. Chem. Mater. } \\
\text { 1998, 10, 3498-3505. }\end{array}$ \\
\hline $\begin{array}{l}p \text {-nitrophenol } \\
\beta \text {-form }\end{array}$ & $\begin{array}{l}\text { high resolution X- } \\
\text { Ray, } 110 \mathrm{~K}\end{array}$ & Single Point & $\begin{array}{l}\text { ELMAM (tyrosine aromatic } \\
\text { ring used to mimic atom- } \\
\text { types) } \\
\text { ELMAM2 }\end{array}$ & $\begin{array}{l}\text { Kulkarni, G. U.; } \\
\text { Kumaradhas, P.; Rao, C. } \\
\text { N. R. Chem. Mater. } \\
\text { 1998, 10, 3498-3505. }\end{array}$ \\
\hline$p$-nitroaniline & X-Ray, 123K & $\begin{array}{l}\text { hydrogen } \\
\text { atoms } \\
\text { positions } \\
\text { optimized }\end{array}$ & ELMAM2 & $\begin{array}{l}\text { Nieger, M. Priv. Comm. } \\
\text { 2007. (NANILI23) }\end{array}$ \\
\hline $\begin{array}{l}p \text {-nitrobenzoic } \\
\text { acid }\end{array}$ & X-Ray, 123K & $\begin{array}{l}\text { hydrogen } \\
\text { atoms } \\
\text { positions } \\
\text { optimized }\end{array}$ & $\begin{array}{l}\text { ELMAM (tyrosine aromatic } \\
\text { ring used to mimic atom- } \\
\text { types) } \\
\text { ELMAM2 }\end{array}$ & $\begin{array}{l}\text { Groth, P. Acta Chem. } \\
\text { Scand. A 1980, 334, 229- } \\
230 .\end{array}$ \\
\hline $\begin{array}{l}\text { 2,5- } \\
\text { dihydroxybenz } \\
\text { oic acid }\end{array}$ & X-Ray, 130K & $\begin{array}{l}\text { hydrogen } \\
\text { atoms } \\
\text { positions } \\
\text { optimized }\end{array}$ & $\begin{array}{l}\text { ELMAM (tyrosine aromatic } \\
\text { ring used to mimic atom- } \\
\text { types) } \\
\text { ELMAM2 }\end{array}$ & $\begin{array}{l}\text { Cohen, D. E.; Benedict, J. } \\
\text { B.; Morlan, B.; Chiu, D. } \\
\text { T.; Kahr, B. Cryst. } \\
\text { Growth Des. 2007, 7, } \\
\text { 492-495. }\end{array}$ \\
\hline $\begin{array}{l}p- \\
\text { dinitrobenzene }\end{array}$ & Neutron, $120 \mathrm{~K}$ & Single Point & $\begin{array}{l}\text { ELMAM (tyrosine aromatic } \\
\text { ring used to mimic atom- } \\
\text { types) } \\
\text { ELMAM2 }\end{array}$ & $\begin{array}{l}\text { Tonogaki, M.; Kawata, } \\
\text { T.; Ohba, S.; Iwata, Y.; } \\
\text { Shibuya, I. Acta } \\
\text { Crystallogr., Sect. B } \\
\text { 1993, 49, 1031-1039. }\end{array}$ \\
\hline $\begin{array}{l}\text { quercetin } \\
\text { monohydrate }\end{array}$ & X-Ray, 100K & $\begin{array}{l}\text { Full } \\
\text { Optimization }\end{array}$ & ELMAM2 & $\begin{array}{l}\text { Domagała, S., Munshi, } \\
\text { P., Ahmed, M., Guillot, } \\
\text { B. \& Jelsch, C. (2011). } \\
\text { Acta Crystallogr., Sect. B } \\
\text { 2011, 67, 63-78. }\end{array}$ \\
\hline
\end{tabular}


Table 4S. Cross-validation statistics for the L-SerVal structure. The average values over the 20 working and test sets are given. All the $R$-factor values are in $\%$.

\begin{tabular}{|lcccc|}
\multicolumn{1}{|c}{ Model } & $w R^{2}(F)$ & $w R^{2}(F)_{\text {free }}$ & $S(F)$ & $S(F)_{\text {free }}$ \\
\hline IAM_U & & & \\
iso & 4.15 & 5.20 & 2.55 & 2.92 \\
\hline IAM_U $_{\text {anis }}$ & 5.10 & 5.33 & 2.62 & 2.99 \\
\hline ELMAM_U $_{\text {iso }}$ & 2.47 & 2.95 & 1.52 & 1.65 \\
\hline ELMAM_U & & & \\
anis & 2.52 & 3.00 & 1.54 & 1.67 \\
\hline ELMAM2_U & 2.53 & 3.07 & 1.55 & 1.71 \\
\hline ELMAM2 & 2.40 & 2.87 & 1.47 & 1.60 \\
\hline
\end{tabular}


Electrostatic Potential quantities computed at the van der Waals surface for tripeptides and aromatic molecules.

All the notations used here to describe the descriptors are the same as in the original papers (Murray \& Politzer, 1998; Murray et al., 2000).

The quantities: $V_{S, \text { max }}, V_{S, \text { min }}, \bar{V}_{S}^{+}, \bar{V}_{S}^{-}, \bar{V}_{S}$ and $\Pi$ are given in $\mathrm{kcal} / \mathrm{mol} ; \sigma_{+}^{2}, \sigma_{-}^{2}$ and $\sigma_{\text {tot }}^{2}$ are in $(\mathrm{kcal} / \mathrm{mol})^{2} ; \quad v$ is dimensionless. $V_{S}^{+}$and $V_{S}^{-}$designate the regions of positive and negative potential respectively. The following definitions were used to calculate surface quantities:

$$
\begin{array}{llrl}
\bar{V}_{S}^{+}=\frac{1}{p} \sum_{i=1}^{p} V_{S}^{+}\left(\mathbf{r}_{i}\right), & \bar{V}_{S}^{-}=\frac{1}{n} \sum_{i=1}^{n} V_{S}^{-}\left(\mathbf{r}_{i}\right), & \overline{V_{S}}=\frac{1}{p+n} \sum_{i=1}^{p+n} V_{S}\left(\mathbf{r}_{i}\right), \\
\Pi=\frac{1}{p+n} \sum_{i=1}^{p+n}\left|V_{S}\left(\mathbf{r}_{i}\right)-\bar{V}_{S}\right|, & \sigma_{+}^{2}=\frac{1}{p} \sum_{i=1}^{p}\left[V_{S}^{+}\left(\mathbf{r}_{i}\right)-\bar{V}_{S}^{+}\right]^{2}, & \sigma_{-}^{2}=\frac{1}{n} \sum_{i=1}^{n}\left[V_{S}^{-}\left(\mathbf{r}_{i}\right)-\bar{V}_{S}^{-}\right]^{2}, \\
\sigma_{\text {tot }}^{2}=\sigma_{+}^{2}+\sigma_{-}^{2}, \quad v=\frac{\sigma_{+}^{2} \sigma_{-}^{2}}{\left(\sigma_{\text {tot }}^{2}\right)^{2}} . &
\end{array}
$$


Table 5S. Electrostatic Potential quantities computed at the van der Waals surface for glycine and tri-peptides.

\begin{tabular}{|c|c|c|c|c|c|c|c|c|}
\hline $\begin{array}{l}\text { Surface } \\
\text { quantity }\end{array}$ & AMBER & ELMAM & ELMAM2 & THEO & AMBER & ELMAM & ELMAM2 & THEO \\
\hline glycine & & & & & AGA & & & \\
\hline$V_{S, \max }$ & 125.1 & 102.8 & 86.3 & 91.4 & 153.2 & 119.8 & 103.0 & 118.5 \\
\hline$V_{S, \min }$ & -122.3 & -102.0 & -77.9 & -78.9 & -159.2 & -138.9 & -105.8 & -110.0 \\
\hline $\bar{V}_{S}^{+}$ & 59.7 & 52.8 & 44.2 & 45.0 & 61.3 & 49.7 & 40.4 & 49.8 \\
\hline $\bar{V}_{S}^{-}$ & -63.5 & -57.5 & -44.7 & -46.3 & -63.2 & -62.9 & -49.3 & -49.9 \\
\hline $\bar{V}_{S}$ & 1.9 & 2.1 & 4.7 & 5.1 & -2.9 & -6.6 & -0.9 & -0.6 \\
\hline$\Pi$ & 61.4 & 54.8 & 44.0 & 45.0 & 62.2 & 56.5 & 44.6 & 49.8 \\
\hline$\sigma_{+}^{2}$ & 1055.0 & 566.7 & 467.5 & 477.8 & 1360.4 & 810.7 & 510.8 & 697.1 \\
\hline$\sigma_{-}^{2}$ & 872.9 & 635.8 & 377.7 & 383.2 & 1611.9 & 1555.3 & 948.2 & 1032.7 \\
\hline$\sigma_{\text {tot }}^{2}$ & 1927.9 & 1202.5 & 845.2 & 861.0 & 2972.3 & 2366.0 & 1459.1 & 1729.8 \\
\hline$v$ & 0.248 & 0.249 & 0.247 & 0.247 & 0.248 & 0.225 & 0.228 & 0.241 \\
\hline$R M S$ & 68.9 & 60.2 & 49.0 & 50.1 & 73.3 & 66.3 & 52.1 & 57.9 \\
\hline$R M S D$ & 20.7 & 12.9 & 2.8 & & 20.4 & 15.4 & 8.9 & \\
\hline$r$ & 0.992 & 0.992 & 0.999 & & 0.979 & 0.982 & 0.992 & \\
\hline$\Delta R / R$ & 0.352 & 0.234 & 0.058 & & 0.314 & 0.248 & 0.163 & \\
\hline
\end{tabular}

\begin{tabular}{|lrrrrrrrr|}
\hline \multicolumn{2}{|c}{$\mathbf{A G A}$} & \multicolumn{1}{c}{$\mathbf{H}_{2} \mathbf{O}$} & & \multicolumn{7}{c|}{$\mathbf{A P A}$} & \multicolumn{1}{c|}{$\mathbf{H}_{\mathbf{2}} \mathbf{O}$} \\
\hline$V_{S, \text { max }}$ & 164.1 & 125.9 & 109.5 & 103.9 & 169.4 & 121.7 & 102.9 & 107.9 \\
\hline$V_{S, \text { min }}$ & -164.4 & -130.4 & -101.2 & -94.0 & -164.4 & -130.8 & -102.7 & -99.8 \\
\hline $\bar{V}_{S}^{+}$ & 68.8 & 57.8 & 48.8 & 46.6 & 62.2 & 48.3 & 40.9 & 40.0 \\
\hline $\bar{V}_{S}^{-}$ & -62.3 & -53.4 & -39.3 & -36.0 & -61.8 & -55.7 & -42.0 & -41.0 \\
\hline $\bar{V}_{S}$ & 0.9 & -2.0 & 2.8 & 4.0 & 0.0 & -4.2 & 1.5 & 2.0 \\
\hline$\Pi$ & 65.5 & 55.3 & 44.0 & 41.3 & 62.0 & 52.1 & 41.4 & 40.4 \\
\hline$\sigma_{+}^{2}$ & 1704.8 & 953.1 & 574.4 & 513.0 & 1796.3 & 822.8 & 556.3 & 549.3 \\
\hline$\sigma_{-}^{2}$ & 1556.8 & 1038.3 & 746.6 & 632.7 & 1553.1 & 963.8 & 667.2 & 586.5 \\
\hline$\sigma_{\text {tot }}^{2}$ & 3261.7 & 1991.4 & 1321.0 & 1145.7 & 3349.4 & 1786.6 & 1223.5 & 1135.9 \\
\hline$V$ & 0.249 & 0.250 & 0.246 & 0.247 & 0.249 & 0.248 & 0.248 & 0.250 \\
\hline $\boldsymbol{R M S}$ & 76.9 & 63.8 & 51.1 & 47.7 & 74.3 & 60.0 & 48.2 & 46.9 \\
\hline
\end{tabular}




\begin{tabular}{|lrrrrrrr|}
\hline $\boldsymbol{R M S D}$ & 32.2 & 20.4 & 6.7 & 31.0 & 17.9 & 4.4 \\
\hline $\boldsymbol{r}$ & 0.976 & 0.980 & 0.993 & 0.971 & 0.981 & 0.996 \\
\hline$\Delta \boldsymbol{R} / \boldsymbol{R}$ & 0.531 & 0.369 & 0.136 & 0.525 & 0.337 & 0.092 \\
\hline
\end{tabular}

\begin{tabular}{|c|c|c|c|c|}
\hline AYA & EtOH & & & \\
\hline$V_{S, \text { max }}$ & 165 & 126.6 & 105.6 & 120.6 \\
\hline$V_{S, \min }$ & -159.7 & -124.1 & -97.1 & -91.5 \\
\hline $\bar{V}_{S}^{+}$ & 56.1 & 46.9 & 35.5 & 44.6 \\
\hline $\bar{V}_{S}^{-}$ & -47.8 & -44 & -33.8 & -32 \\
\hline $\bar{V}_{S}$ & -1.1 & -2.9 & 1.7 & -0.7 \\
\hline$\Pi$ & 51.4 & 45.1 & 34.6 & 37 \\
\hline$\sigma_{+}^{2}$ & 1840.6 & 1143.2 & 693.7 & 1013.7 \\
\hline$\sigma_{-}^{2}$ & 1419.1 & 884.8 & 605.6 & 540.5 \\
\hline$\sigma_{\text {tot }}^{2}$ & 3259.7 & 2028 & 1299.3 & 1554.2 \\
\hline$v$ & 0.246 & 0.246 & 0.249 & 0.227 \\
\hline$R M S$ & 65.4 & 55.3 & 43.0 & 46.4 \\
\hline RMSD & 24.3 & 14.8 & 9.7 & \\
\hline$r$ & 0.963 & 0.974 & 0.981 & \\
\hline$\Delta R / R$ & 0.441 & 0.292 & 0.216 & \\
\hline all & peptides & & & \\
\hline$<R M S>$ & 71.8 & 61.1 & 48.7 & 49.8 \\
\hline$<R M S D>$ & 25.7 & 16.3 & 6.5 & \\
\hline$<r>$ & 0.976 & 0.982 & 0.992 & \\
\hline$<\Delta R / R>$ & 0.433 & 0.296 & 0.133 & \\
\hline
\end{tabular}


Table 6S. Computed Electrostatic Potential surface quantities for the aromatic molecules.

\begin{tabular}{|c|c|c|c|c|c|c|}
\hline Surface quantity & ELMAM & ELMAM2 & THEO & ELMAM & ELMAM2 & THEO \\
\hline benzene & & & & & & \\
\hline$V_{S, \max }$ & 39.3 & 20.5 & 18.9 & 62.7 & 62.9 & 60.2 \\
\hline$V_{S, \min }$ & -56.2 & -36.5 & -27.2 & -59.6 & -45.0 & -36.3 \\
\hline $\bar{V}_{S}^{+}$ & 16.2 & 10.9 & 9.4 & 23.5 & 18.7 & 16.1 \\
\hline $\bar{V}_{S}^{-}$ & -24.9 & -15.1 & -13.3 & -21.9 & -18.3 & -15.1 \\
\hline $\bar{V}_{S}$ & -5.2 & 0.2 & 0.3 & -2.2 & 0.7 & 1.0 \\
\hline$\Pi$ & 20.8 & 12.6 & 10.9 & 22.4 & 18.5 & 15.6 \\
\hline$\sigma_{+}^{2}$ & 87.5 & 21.3 & 15.6 & 209.5 & 122.7 & 103.8 \\
\hline$\sigma_{-}^{2}$ & 212.2 & 94.6 & 66.9 & 156.6 & 108.2 & 68.1 \\
\hline$\sigma_{\text {tot }}^{2}$ & 299.7 & 115.9 & 82.5 & 366.0 & 230.9 & 171.9 \\
\hline$v$ & 0.207 & 0.150 & 0.153 & 0.245 & 0.249 & 0.239 \\
\hline$R M S$ & 24.5 & 14.7 & 12.6 & 26.3 & 21.4 & 18.2 \\
\hline RMSD & 13.5 & 2.4 & & 11.7 & 6.0 & \\
\hline$r$ & 0.961 & 0.995 & & 0.935 & 0.967 & \\
\hline$\Delta R / R$ & 0.767 & 0.178 & & 0.536 & 0.305 & \\
\hline
\end{tabular}

\begin{tabular}{|c|c|c|c|c|c|c|}
\hline resorcinol & & & & & & \\
\hline$V_{S, \max }$ & 58.7 & 61.0 & 55.4 & 67.9 & 68.0 & 63.8 \\
\hline$V_{S, \min }$ & -58.1 & -42.3 & -37.4 & -55.1 & -38.4 & -35.9 \\
\hline $\bar{V}_{S}^{+}$ & 26.3 & 22.8 & 21.0 & 23.6 & 16.4 & 18.2 \\
\hline $\bar{V}_{S}^{-}$ & -23.9 & -19.3 & -19.0 & -17.6 & -12.2 & -14.0 \\
\hline $\bar{V}_{S}$ & -1.5 & 1.3 & 0.4 & 0.7 & 2.8 & 2.3 \\
\hline$\Pi$ & 24.9 & 21.1 & 20.0 & 20.4 & 14.4 & 16.2 \\
\hline$\sigma_{+}^{2}$ & 223.9 & 123.4 & 112.7 & 254.8 & 158.9 & 157.9 \\
\hline$\sigma_{-}^{2}$ & 174.5 & 104.9 & 87.9 & 134.5 & 76.5 & 75.5 \\
\hline$\sigma_{\text {tot }}^{2}$ & 398.4 & 228.3 & 200.6 & 389.3 & 235.4 & 233.3 \\
\hline$v$ & 0.246 & 0.248 & 0.246 & 0.226 & 0.219 & 0.219 \\
\hline$R M S$ & 28.7 & 23.7 & 22.3 & 24.7 & 18.2 & 19.6 \\
\hline RMSD & 10.0 & 5.1 & & 9.2 & 5.7 & \\
\hline
\end{tabular}




\begin{tabular}{|lllll|}
\hline $\boldsymbol{r}$ & 0.955 & 0.978 & 0.944 & 0.957 \\
\hline$\Delta \boldsymbol{R} / \boldsymbol{R}$ & 0.400 & 0.222 & 0.417 & 0.302 \\
\hline
\end{tabular}

\begin{tabular}{|c|c|c|c|c|}
\hline quercetin & & & & \\
\hline$V_{S, \max }$ & 72.8 & 76.9 & 80.2 & 68.4 \\
\hline$V_{S, \min }$ & -60.4 & -44.9 & -50.5 & -51.2 \\
\hline $\bar{V}_{S}^{+}$ & 18.4 & 19.6 & 32.3 & 24.7 \\
\hline $\bar{V}_{S}^{-}$ & -14.2 & -13.7 & -25.9 & -22.1 \\
\hline $\bar{V}_{S}$ & 3.4 & 2.8 & 1.3 & 3.3 \\
\hline$\Pi$ & 16.5 & 16.8 & 29.0 & 23.3 \\
\hline$\sigma_{+}^{2}$ & 197.1 & 208.7 & 434.4 & 222.9 \\
\hline$\sigma_{-}^{2}$ & 158.2 & 113.0 & 157.9 & 230.2 \\
\hline$\sigma_{\text {tot }}^{2}$ & 355.2 & 321.7 & 592.4 & 453.0 \\
\hline$v$ & 0.247 & 0.228 & 0.196 & 0.250 \\
\hline$R M S$ & 21.3 & 21.1 & 33.7 & 27.9 \\
\hline RMSD & 6.7 & & 10.3 & \\
\hline$r$ & 0.950 & & 0.964 & \\
\hline$\Delta R / R$ & 0.316 & & 0.335 & \\
\hline
\end{tabular}

\begin{tabular}{|c|c|c|c|c|c|c|c|}
\hline nitrophenol & alpha & & & Nitrophenol & beta & & \\
\hline$V_{S, \max }$ & 97.5 & 76.4 & 71.7 & & 101.8 & 78.4 & 77.5 \\
\hline$V_{S, \min }$ & -58.1 & -32.2 & -42.0 & & -57.6 & -32.1 & -41.2 \\
\hline $\bar{V}_{S}^{+}$ & 29.3 & 20.3 & 18.2 & & 29.1 & 20.5 & 17.8 \\
\hline $\bar{V}_{S}^{-}$ & -26.5 & -12.8 & -18.6 & & -26.7 & -12.8 & -17.9 \\
\hline $\bar{V}_{S}$ & -1.3 & 3.5 & 4.5 & & -1.3 & 3.4 & 4.4 \\
\hline$\Pi$ & 27.7 & 16.8 & 17.7 & & 27.7 & 16.8 & 17.2 \\
\hline$\sigma_{+}^{2}$ & 477.3 & 209.1 & 178.1 & & 493.3 & 214.0 & 196.2 \\
\hline$\sigma_{-}^{2}$ & 183.8 & 69.8 & 156.1 & & 181.8 & 69.2 & 154.2 \\
\hline$\sigma_{\text {tot }}^{2}$ & 661.1 & 279.0 & 334.2 & & 675.1 & 283.2 & 350.4 \\
\hline$v$ & 0.201 & 0.188 & 0.249 & & 0.197 & 0.185 & 0.246 \\
\hline$R M S$ & 33.0 & 20.6 & 22.5 & & 33.1 & 20.7 & 22.3 \\
\hline RMSD & 21.6 & 6.0 & & & 21 & 5.8 & \\
\hline$r$ & 0.786 & 0.964 & & & 0.806 & 0.966 & \\
\hline
\end{tabular}




\begin{tabular}{|c|c|c|}
\hline$\Delta \boldsymbol{R} / \boldsymbol{R}$ & 0.280 & 0.772 \\
\hline
\end{tabular}

\begin{tabular}{|c|c|c|c|c|c|c|}
\hline$p$-nitrobenzoic & acid & & & & & \\
\hline$V_{S, \max }$ & 56.6 & 48.6 & 47.7 & 59.2 & 48.1 & 49.5 \\
\hline$V_{S, \min }$ & -36.2 & -32.0 & -37.4 & -37.2 & -22.4 & -30.7 \\
\hline $\bar{V}_{S}^{+}$ & 23.2 & 15.3 & 16.3 & 23.0 & 16.5 & 22.2 \\
\hline $\bar{V}_{S}^{-}$ & -13.2 & -13.4 & -18.2 & -12.0 & -10.9 & -16.1 \\
\hline $\bar{V}_{S}$ & 0.6 & 4.2 & 5.4 & 0.9 & 5.9 & 7.7 \\
\hline$\Pi$ & 17.1 & 14.1 & 15.3 & 16.3 & 13.4 & 18.4 \\
\hline$\sigma_{+}^{2}$ & 207.1 & 117.6 & 99.4 & 258.5 & 103.8 & 91.0 \\
\hline$\sigma_{-}^{2}$ & 57.2 & 72.3 & 84.2 & 90.8 & 30.0 & 56.1 \\
\hline$\sigma_{\text {tot }}^{2}$ & 264.2 & 189.9 & 183.6 & 349.3 & 133.9 & 147.1 \\
\hline$v$ & 0.17 & 0.236 & 0.248 & 0.192 & 0.174 & 0.236 \\
\hline$R M S$ & 20.6 & 17.7 & 19.5 & 20.9 & 15.9 & 21.9 \\
\hline RMSD & 17.2 & 8.2 & & 24.1 & 6.7 & \\
\hline $\boldsymbol{r}$ & 0.652 & 0.900 & & 0.376 & 0.969 & \\
\hline$\Delta R / R$ & 0.857 & 0.444 & & 1.126 & 0.358 & \\
\hline
\end{tabular}

\begin{tabular}{|lrrr|}
\hline All aromatic & & & \\
\hline$<\boldsymbol{R M S}>$ & 26.5 & 20.8 & 20.8 \\
\hline$<\boldsymbol{R M S D}>$ & 16.0 & 6.3 & \\
\hline$<\boldsymbol{r}>$ & 0.802 & 0.961 & \\
\hline$<\Delta \boldsymbol{R} / \boldsymbol{R}>$ & 0.708 & 0.301 \\
\hline
\end{tabular}


(a)

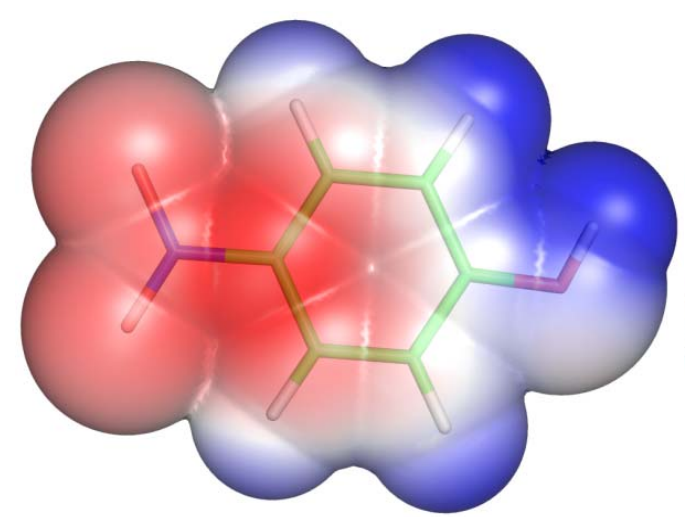

(c)

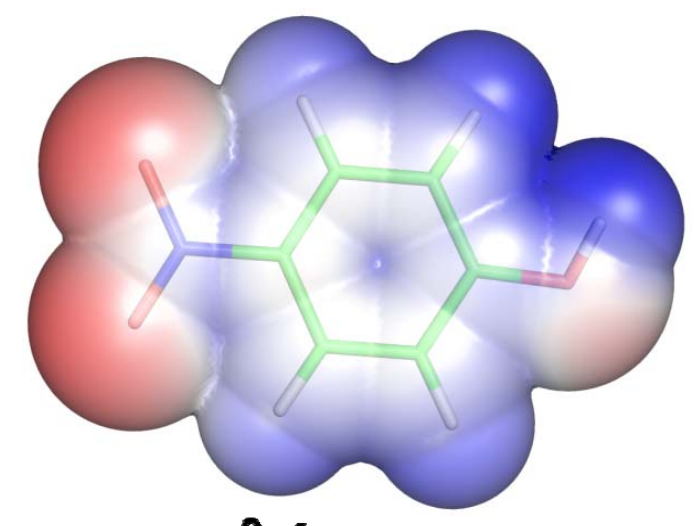

$-0.15 e^{-1}$ (b)

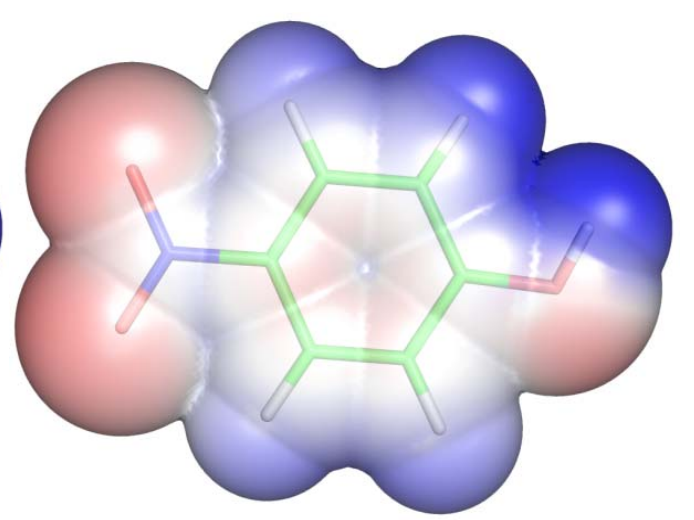

$0.15 \mathrm{eA}^{-1}$

Figure 1S. The electrostatic potential mapped the van der Waals surface in the nitrophenol (alpha) molecule for (a) ELMAM (b) ELMAM2 and (c) THEO_CRYS models. The maximum negative (blue) and positive (red) values of the ESP correspond to -0.15 and $0.15 \mathrm{e}^{-1}$ values, respectively. The $\Delta V / V$ values are 0.791 and 0.280 for ELMAM and ELMAM2, respectively. 


\section{References of molecules used for the ELMAM2 Database construction}

Benabicha, F., Pichon-Pesme, V., Jelsch, C., Lecomte, C. \& Khmou, A. (2000). Acta Cryst. B56, 155165.

Birkedal, H., Madsen, D., Mathiesen, R. H., Knudsen, K., Weber, H.-P., Pattisond, P. \&

Schwarzenbach, D. (2004). Acta Cryst. A60, 371-381.

Bouhmaida, N., Bonhomme, F., Guillot, B., Jelsch, C. \& Ghermani, N. E. (2009). Acta Cryst. B65, 363-374.

Chen, Y-S., Stash, A. I. \& Pinkerton, A. A. (2007). Acta Cryst. B63, 309-318.

Coppens, P., Abramov, Y., Carducci, M., Korjov, B., Novozhilova, I., Alhambra, C. \& Pressprich, M. R. (1999). J. Am. Chem. Soc. 121, 2585-2593.

Dahaoui, S., Jelsch, C., Howard, J. A. K. \& Lecomte, C. (1999). Acta Cryst. B55, 226-230.

Destro, R., Marsh, R. E. \& Bianchi, R. (1988). J. Phys. Chem. 92, 966-973.

Dittrich, B., Koritsánszky, T., Grosche, M., Scherer, W., Flaig, R., Wagner, A., Krane, H. G., Kessler, H., Riemer, C., Schreurs, A. M. M., \& Luger, P. (2002). Acta Cryst. B58, 721-727.

Dittrich, B., Munshi, P. \& Spackman, M. A. (2007). Acta Cryst. B63, 505-509.

Dittrich, B. \& Spackman, M. A. (2007). Acta Cryst. A63, 426-436.

Domagała, S., Korybut-Daszkiewicz, B., Straver, L., \& Woźniak, K., (2009). Inorg. Chem. 48, 4010 4020.

Dominiak, P. M., Grech, E., Barr, G., Teat, S., Mallinson, P. \& Woźniak, K., (2003). Chem. Eur. J. 9, 963-970.

Espinosa, E., Lecomte, C., Molins, E., Veintemillas, S., Cousson, A. \& Paulus, W. (1996). Acta Cryst. B52, 519-534.

Fournier, B., Bendeif, E-E., Guillot, B., Podjarny, A., Lecomte, C. \& Jelsch, C. (2009). J. Am. Chem. Soc. 131, 10929-10941.

Ghermani, N. E., Spasojević-de Biré, A., Bouhmaida, N., Ouharzoune, S., Bouligand, J., Layre, A., Gref, R. \& Couvreur, P. (2004). Pharmaceutical Research, 21, 598-607.

Guillot, B., Muzet, N., Artacho, E., Lecomte, C. \& Jelsch, C. (2003). J. Phys. Chem. B, 107, 91099121.

Howard, J. A. K., Mahon, M. F., Raithby, P. R. \& Sparkes, H. A. (2009). Acta Cryst. B65, 230-237. Hübschle, C. B., Dittrich, B., Grabowsky, S., Messerschmidt, M. \& Luger, P. (2008). Acta Cryst. B64, 363-374.

Kalinowski, R., Dittrich, B., Hübschle, C. B., Paulmann, C. \& Luger, P. (2007). Acta Cryst. B63, 753-767.

Luger, P., Messerschmidt, M., Scheins, S. \& Wagner, A. (2004). Acta Cryst. A60, 390-396.

Lutz, M., Spek, A. L., van der Geer, E. P. L., van Koten, G. \& Gebbink, R. J. M. K. (2008). Acta Cryst. C64, o87-090.

Madsen, A. Ø., Sørensen, H. O., Flensburg, C., Stewart, R. F. \& Larsen, S. (2004). Acta Cryst. A60, 550-561.

Martin, A. \& Pinkerton, A. A. (1998). Acta Cryst. B54, 471-477.

Meents, A., Dittrich, B., Johnas, S. K. J., Thome, V. \& Weckert, E. F. (2008). Acta Cryst. B64, 42-49.

Munshi, P. \& Guru Row, T. N. (2002). Acta Cryst. B58, 1011-1017.

Munshi, P. \& Guru Row, T. N. (2005). J. Phys. Chem. A, 109, 659-672.

Munshi, P. \& Guru Row, T. N. (2006a). Cryst. Growth \& Des. 6, 708-718. 
Munshi, P. \& Guru Row, T. N. (2006b). Acta Cryst. B62, 612-626.

Munshi, P., Thakur, T. S., Guru Row, T. N. \& Desiraju, G. R. (2006c). Acta Cryst. B62, 118-127.

Ogawa, K., Noda, Y., Lüthi, T. \& Buergi, H.-B. (2006). Priv. Comm.

Overgaard, J. \& Hibbs, D. E. (2004). Acta Cryst. A60, 480-487.

Pichon-Pesme, V., Lachekar, H., Souhassou, M. \& Lecomte, C. (2000). Acta Cryst. B56, 728-737.

Parrish, D., Zhurova, E. A., Kirschbaum, K. \& Pinkerton, A. A. (2006). J. Phys. Chem. B, 110, $26442-$ 26447.

Rodrigues, B. L., Tellgren, R. \& Fernandes, N. G. (2001). Acta Cryst. B57, 353-358.

Scheins, S., Dittrich, B., Messerschmidt, M., Paulmann, C. \& Luger, P. (2004). Acta Cryst. B60, 184190.

Slouf, M., Holy, A., Petricek, V. \& Cisarova, I. (2002). Acta Cryst. B58, 519-529.

Sørensen, H. O., Stewart, R. F., McIntyre, G. J. \& Larsen, S. (2003). Acta Cryst. A59, 540-550.

Sparkes, H. A., Brayshaw, S. K., Weller, A. S. \& Howard, J. A. K. (2008). Acta Cryst. B64, 550-557.

Volkov, A., Abramov, Y., Coppens, P. \& Gatti C. (2000). Acta Cryst. A56, 332-339.

Wiest, R., Pichon-Pesme, V., Bénard, M. \& Lecomte, C. (1994). J. Phys. Chem. 98, 1351-1362.

Zhurov, V. V., Zhurova, E. A., Chen Y-S. \& Pinkerton, A. A. (2005). J. Appl. Cryst. 38, 827-829.

Zhurova, E. A., Martin, A. \& Pinkerton, A. A. (2002) J. Am. Chem. Soc. 124, 8741-8750.

Zhurova, E. A., Matta, C. F., Wu, N., Zhurov, V. V. \& Pinkerton, A. A. (2006). J. Am. Chem. Soc. 128, 8849-8861.

Zhurova, E. A. \& Pinkerton, A. A. (2001). Acta Cryst. B57, 359-365. 